Relato de Experiência | Experience Report

\title{
Reflexiones desde las Terapias Ocupacionales desde el Sur: violencia estructural, derechos humanos y género en procesos de acompańamiento de mujeres durante el embarazo y maternaje ${ }^{1}$
}

Reflexóes desde as Terapias Ocupacionais do Sul: violência estrutural, direitos humanos e gênero nos processos de acompanhamento de mulheres durante a gravidez e a maternidade

Reflections from Occupational Therapies from the South: structural violence, human rights and gender in processes of accompaniment of women during pregnancy and mothering

Mónica Díaz (D), Tamara Palomino a,b,c (D), Natalia Quintana ${ }^{\mathrm{a}, \mathrm{b}}$ (D), Mónica Palacios ${ }^{\mathrm{a}}$ (D), Paulina Aracena ${ }^{\mathrm{b}}$

a Universidad de Santiago de Chile - USACH, Santiago, Chile.

${ }^{b}$ Hospital y Centro de Referencia en Salud El Pino, Santiago, Chile

${ }^{c}$ Centro Espacio Seguro, Santiago, Chile

Cómo citar: Díaz, M., Palomino, T., Quintana, N., Palacios, M., \& Aracena, P. (2021). Reflexiones desde las Terapias Ocupacionales desde el Sur: violencia estructural, derechos humanos y género en procesos de acompañamiento de mujeres durante el embarazo y maternaje. Cadernos Brasileiros de Terapia Ocupacional, 29, e2996. https://doi.org/10.1590/2526-8910.ctoRE2270

\begin{abstract}
$\underline{\text { Resumen }}$
Se trata de un relato de experiencias que surge a partir de una investigación en curso que caracteriza el modelo de intervención de un Programa Ambulatorio Intensivo de tratamiento para el consumo de drogas y/o alcohol específico para mujeres adultas en embarazo o puerperio del Hospital El Pino, en Santiago de Chile. El objetivo es adelantar algunas reflexiones a partir de esta experiencia investigativa en curso focalizando en las formas de acompańamiento que el equipo de terapeutas ocupacionales realiza en este programas. Se presentan los antecedentes, elementos teóricos que guían esta investigación, los procedimientos, y las reflexiones en torno a los procesos de acompañamiento que realiza el equipo de terapia ocupacional
\end{abstract}

\footnotetext{
${ }^{1}$ Este artículo es parte del proyecto de investigación "Modelo de Intervención con Enfoque de Género Programa Ambulatorio Intensivo de alcohol y/o drogas para mujeres en embarazo y/o post parto”. Proyecto InvClínica_DICYT, Código 022091PT_MED.
} 
considerando los análisis cualitativos preliminares. Las primeras acciones de investigación en torno a esta experiencia nos muestran que los procesos de acompañamiento en terapia ocupacional situados desde perspectivas descoloniales, permiten una lectura político-teórica con base en los derechos humanos y perspectiva de género, visibilizando la violencia estructural producida por el sistema colonial/moderno/patriarcal/ racial que atraviesa las historias de vida de las mujeres y sus familias. Es fundamental garantizar los derechos de las mujeres, articular acciones en los espacios de vida cotidiana y fortalecer las diversas redes de soporte y apoyo para la reconstrucción de sus proyectos de vida. Se concluye que el consumo de drogas, la experiencia de embarazo y el maternaje son condicionados por las desigualdades, estigmatización y violencia estructural del sistema moderno colonial por lo cual es muy relevante que los acompańamientos en terapia ocupacional incorporen perspectivas descoloniales desde el sur.

Palabras-clave: Terapia Ocupacional, Derechos Colectivos, Construcción Social del Género, Consumo de drogas, Violencia, Maternidades.

\section{$\underline{\text { Resumo }}$}

É um relato de experiências que surge de uma pesquisa em andamento que caracteriza o modelo de intervençáo de um Programa Ambulatorial Intensivo de tratamento para o consumo de drogas e/ou álcool, específico para mulheres adultas na gravidez ou puerpério, no Hospital El Pino, em Santiago do Chile. O objetivo é avançar em algumas reflexōes a partir dessa experiência de pesquisa em andamento, enfocando as formas de acompanhamento que a equipe de terapeutas ocupacionais realiza nesse programa. São apresentados os antecedentes, os elementos teóricos que norteiam esta pesquisa, os procedimentos e as reflexóes sobre os processos de acompanhamento realizados pela equipe de terapia ocupacional, considerando as análises qualitativas preliminares. As primeiras açóes de pesquisa em torno dessa experiência nos mostram que os processos de acompanhamento em terapia ocupacional, situados a partir de perspectivas decoloniais, permitem uma leitura teórico-política baseada nos direitos humanos e de gênero, tornando visíveis as violências estruturais produzidas pelo sistema colonial / moderno / patriarcal / racial, que perpassa as histórias de vida de mulheres e de suas famílias. É imprescindível garantir os direitos das mulheres, articular açóes nos espaços da vida cotidiana e fortalecer as diversas redes de apoio e apoio à reconstruçáo de seus projetos de vida. Conclui-se que o uso de drogas, a vivência da gravidez e da maternidade são condicionados pelas desigualdades, estigmatizaçóes e violências estruturais do sistema colonial moderno, por isso é muito relevante que os acompanhamentos em terapia ocupacional incorporem perspectivas decoloniais do sul.

Palavras-chave: Terapia Ocupacional, Direitos Coletivos, Construçáo Social do Gênero, Consumo de Drogas, Violência, Maternalidades.

\section{$\underline{\text { Abstract }}$}

It is a report of experiences that arises from an ongoing investigation that characterizes the intervention model of an Intensive Outpatient Program of treatment for drug and/or alcohol consumption specific for adult women in pregnancy or puerperium at Hospital El Pino, in Santiago de Chile. The objective is to advance some reflections based on this ongoing research experience, focusing on the forms of accompaniment that the team of occupational therapists carries out in these programs. The 
antecedents, theoretical elements that guide this research, the procedures, and the reflections on the accompaniment processes carried out by the occupational therapy team are presented, considering the preliminary qualitative analyzes. The first research actions around this experience show us that the accompaniment processes in occupational therapy located from decolonial perspectives, allow a political-theoretical reading based on human rights and gender, making visible the structural violence produced by the colonial system/modern/patriarchal/racial running through the life stories of women and their families. It is essential to guarantee the rights of women, to articulate actions in the spaces of daily life, and to strengthen the various support and support networks for the reconstruction of their life projects. It is concluded that drug use, the experience of pregnancy, and mothering are conditioned by the inequalities, stigmatization, and structural violence of the modern colonial system, which is why it is very relevant that the accompaniments in occupational therapy incorporate decolonial perspectives from the south.

Keywords: Occupational Therapy, Equal Rights, Social Construction of Gender, Substance Abuse, Violence, Maternity.

\section{Escenario de la Experiencia}

El Programa ambulatorio intensivo para el consumo de drogas y/o alcohol específico para mujeres en embarazo o puerperio (en adelante PAI-M) del Hospital y Centro de Referencia en Salud El Pino (en adelante CRS El Pino), situado en la comuna de San Bernardo, Chile, atiende a mujeres con similares características sociodemográficas que las encontradas a nivel nacional en la población que recibe atención, entre ellas: $75 \%$ son solteras, viven de allegadas y no cuentan con un trabajo remunerado, $40 \%$ tiene educación media incompleta y el 25\% no terminó la educación básica (Rowlands et al., 2018). No obstante, con este grupo de mujeres, el PAI-M del CRS El Pino presenta porcentajes de adherencia y alta terapéutica por encima de la media a nivel de Región Metropolitana, que se expresa en los siguientes resultados del 2018: 25\% de abandono, 41\% de alta terapéutica, $24 \%$ derivación, $10 \%$ alta administrativa, obteniendo un total de $35 \%$ de tratamientos interrumpidos (abandono más alta administrativa), cifra que dista notablemente del $66 \%$ obtenido a nivel de la Región Metropolitana (Servicio Nacional para la Prevención y Rehabilitación del Consumo de Drogas y Alcohol, 2018). La comuna de San Bernardo presenta una pobreza multidimensional del $22 \%$, existiendo un número importante de personas que carecen de servicios básicos y viven en condiciones de hacinamiento (Ministerio de Desarrollo Social, 2016).

Los PAI-M, se implementaron el año 2005, como plan piloto producto de la evidencia de que un problema de consumo de drogas y/o alcohol no presenta las mismas consecuencias físicas, psicológicas ni sociales en mujeres, siendo un grupo que requiere intervenciones terapéuticas diferenciadas, que consideren aspectos contextuales y socioculturales que complejizan la situación de consumo en población femenina (ValenciaRecabarren, 2015). En el caso específico de mujeres en situación de embarazo o puerperio, se ha identificado que la mayoría de los recursos terapéuticos tiene un abordaje genérico centrado en la rehabilitación de drogas, sin considerar la condición de mujer y maternidad, tampoco incluyen el contexto socio-familiar, considerando que es "[...] frecuente la 
existencia de una pareja consumidora, o bien de una historia de violencia doméstica o situación de maltrato o abuso en la niñez y adolescencia [...]" (Pascale, 2010, p. 5); por lo cual se hace necesario un abordaje situacional específico capaz de integrar dicha complejidad. Entre las particularidades de la situación, se señala que un número importante de embarazadas "[...] son reticentes a manifestar el consumo de sustancias psicoactivas por miedo a perder la custodia de sus hijos, por tener otros problemas legales y en algunos casos por la "mirada" del personal de la salud, que aleja a la usuaria de los dispositivos sanitarios [...]" (Pascale, 2010, p. 56). Esto hace necesario, que los equipos generen modelos de gestión capaces de incorporar estos aspectos diferenciales que están presentes en muchas mujeres en situación de embarazo y puerperio y asuman la figura de garantes de derecho que les compete (Ministerio de Salud, 2016; Servicio Nacional para la Prevención y Rehabilitación del Consumo de Drogas y Alcohol, 2018). Como menciona Lemus (2013), una situación de consumo problemático en una mujer representa un reto a los estereotipos culturales y sociales asociados a lo femenino, generando una contradicción con el rol tradicionalmente esperado; es por esto que son víctimas de mayores niveles de estigmatización, asociada, entre otras cosas, al supuesto abandono o mala práctica del "rol materno". Para aquellas mujeres que se encuentran embarazadas los niveles de estigmatización se agudizan aún más, por cuanto "[...] el consumo de sustancias durante la gestación se ha relacionado con consecuencias negativas para la salud del binomio materno fetal, poniendo en riesgo la vida de la madre y su hijo [...]" (Pascale, 2010, p. 5). Sumado a lo anterior, se encuentran los efectos en sus familias y en su entorno comunitario (Servicio Nacional para la Prevención y Rehabilitación del Consumo de Drogas y Alcohol, 2018).

En la Guía Técnica Mujeres y Tratamiento de Drogas, del año 2016, se puntualiza que en general las mujeres muestran un mayor nivel de motivación para el tratamiento que los hombres, pero tienen índices más bajos de permanencia (Servicio Nacional para la Prevención y Rehabilitación del Consumo de Drogas y Alcohol, 2018), siendo esto parte del problema. Esto debido a que los programas de tratamiento en drogas han sido históricamente pensados para hombres, existiendo poco desarrollo e implementación de estrategias de intervención que incorporen de forma situada un enfoque de género que dé respuesta a los obstaculizadores de su permanencia (Lorenzo, 2009) El abordaje del consumo de drogas y alcohol y sus modelos de gestión tienden a homogeneizar las intervenciones sin distinciones de género ni distinciones locales. Según Consejo Nacional para el Control de Estupefacientes (2009) es importante considerar la situación específica que se presenta en este grupo, indicando que, entre las embarazadas con consumo de drogas, hay un inicio tardío de los controles prenatales, se asocia a violencia de género en primigestas, dificultad para aceptar el embarazo, ambivalencia u ocultamiento del embarazo por miedo o temor. Así mismo, se explica que la presencia de violencia durante el periodo de gestación incide en la baja autoestima, síntomas depresivos, riesgo para la salud física y aumento de la probabilidad de consumo de sustancias, así como insuficiente adherencia a los cuidados prenatales (Ministerio de Salud, 2008). Con respecto a los efectos en el feto, hijas o hijos, el uso de sustancias psicoactivas en mujeres embarazadas, se constituye en un factor estresante del ambiente intrauterino (Pascale, 2015) y, los síntomas y conductas presentes en niños y niñas (Bobes et al., 2011). Las intervenciones deberían proteger entonces la integridad del niño o niña, mediante la rehabilitación de las madres (Ramírez-Elizondo, 2011).

Actualmente en Chile existen tres documentos que orientan el trabajo con mujeres en programas de tratamiento de consumo problemático (Servicio Nacional para la Prevención 
y Rehabilitación del Consumo de Drogas y Alcohol, 2009, 2016, 2018) entregando elementos teóricos que apoyan las intervenciones, pero en las prácticas de atención queda en evidencia que existe una brecha para llevarlos a cabo de forma efectiva (Nebot et al., 2011). Entre las barreras de acceso a programas de prevención y tratamiento (Servicio Nacional para la Prevención y Rehabilitación del Consumo de Drogas y Alcohol, 2009), se encuentra la detección tardía por omisión del problema debido a la estigmatización vivida por las mujeres, lo que se expresa en un intenso sentimiento de culpa y vergüenza, la falta de horarios flexibles y capacidad de respuesta inmediata por parte de los servicios de salud, terapias no adaptadas a las condiciones de la población con mayor vulnerabilidad a la violencia, la no consideración de necesidades de capacitación y empleo, la falta de atención para hijos e hijas, falta de formación sobre crianza y educación en infancia, consumo de drogas y/o alcohol por parte de su parejas y escaso apoyo de sus familias (Servicio Nacional para la Prevención y Rehabilitación del Consumo de Drogas y Alcohol, 2016).

\section{Desigualdades y Ausencia de Perspectivas de Género y Derechos en los Procesos de Acompańamiento}

Las perspectivas de género y derechos, son centrales para el desarrollo de los PAI-M, frente a las necesidades expuestas por la población de mujeres con condiciones de salud mental complejas, situación de consumo problemático severo, vulneración psicosocial, cesantía, problemáticas cognitivas, multíparas y/o en embarazo y/o post parto. En el caso del enfoque de género la misma Organización Mundial de la Salud (2007) señala al género como orientador de las funciones, comportamientos, actividades y atributos que cada sociedad considera apropiados para hombres y mujeres, los que pueden generar desigualdades que favorecen sistemáticamente a uno de los dos grupos. Una salud pública basada en un enfoque de género reconoce las diferencias entre ellos, lo que nos sirve para comprender las diferencias de resultados, experiencias y riesgos sanitarios entre hombres y mujeres (Organización Mundial de la Salud, 2007). Respecto al consumo problemático de mujeres en contexto de vulnerabilidad, se observan condiciones que complejizan su situación, siendo evidente la diferencia entre un hombre consumidor y una mujer consumidora. Socialmente se acepta con mayor facilidad el consumo de drogas y/o alcohol en hombres que, en mujeres, siendo éstas fuertemente recriminadas (Consejo Nacional para el Control de Estupefacientes, 2007), especialmente cuando son madres. Según Romero \& Medina-Moreno (1996), un problema de adicción en una mujer representa un reto a los estereotipos culturales y sociales definidos como expectativas aceptables o normales en relación a la feminidad, constituyéndose una amenaza al rol femenino tradicional, considerando que han perdido respetabilidad. Cuando la mujer presenta un consumo problemático, generalmente es el espacio cotidiano- privado, el que se ve afectado: labores domésticas quedan sin realizar y el cuidado de hijos e hijas se ve menoscabado, así la mujer comienza a alejarse del rol históricamente asignado, como las labores que debe cumplir en su hogar. Su situación es percibida como fuera de control, impactando en su confiabilidad e imagen social, primando en ellas, sentimientos de vergüenza y culpa. Generalmente el hombre no asume responsabilidad en los quehaceres hogareńos, ya que sus funciones sociales se mantienen en el espacio público. En los hombres, la normalización del consumo es comprendida y aceptada como perteneciente a su mundo, siendo señal de masculinidad (Lemus, 2013). Sobre las características socio demográficas de esta población, se puede observar que al comparar al grupo en tratamiento por consumo problemático de drogas y/o alcohol de 
mujeres en embarazo y postparto con las mujeres no embarazadas, las primeras muestran signos de mayor nivel de deterioro social. El 51\% de las mujeres no han completado la enseñanza media, en el caso de las mujeres en embarazo y postparto la cifra asciende al 64.4\%; el 31\% de las mujeres tiene enseńanza básica o menos, para las mujeres en embarazo y postparto este dato es del 39.4\%. Cuando se estima la condición laboral, el 30\% de las mujeres en tratamiento tiene un trabajo remunerado, este dato es de solo $17.7 \%$ para las mujeres en embarazo y postparto; el $30 \%$ de las mujeres en tratamiento están cesantes y en el caso de las mujeres en embarazo y postparto el porcentaje es de $33.7 \%$; el $21 \%$ y el $26.4 \%$ se dedica a los quehaceres de hogar, respectivamente (Servicio Nacional para la Prevención y Rehabilitación del Consumo de Drogas y Alcohol, 2016). Las desigualdades referidas se constituyen en vulneración de derechos hacia las mujeres en diversas dimensiones.

Con respecto al enfoque de derechos, es fundamental el reconocimiento de las mujeres como titulares de derecho en el marco de un sistema colonial-patriarcal que atraviesa sus historias de vida. Esto, implica problematizar la violencia estructural contra las mujeres como un asunto transversal (Parra \& Tortosa, 2003; Follegati et al., 2019; Segato, 2003) que afecta sus condiciones concretas de existencia, el acceso a derechos (Artigas, 2005; Guajardo \& Galheigo, 2015) sus subjetividades y sus posibilidades de recrear sus proyectos en un contexto histórico determinado que les permita producir su existencia (Lopes et al., 2014). La violencia estructural considera la violencia más allá del daño físico o directo y vislumbra "[...] la existencia de una estratificación social que da origen a una forma de reparto desfavorable para las mujeres [...]” (Follegati et al., 2019, p. 21). En el caso del consumo y de la violencia específica que se engendra de esta situación, las mujeres han vivido diversos tipos de violencia estructural con respecto al acceso a sus derechos y libertades. Esta violencia se hace presente incluso al interior de sus núcleos familiares, existiendo situaciones de abuso y abandono, producto de historias familiares, que han venido reproduciendo el daño transgeneracional y la precariedad de sus vidas.

Las mujeres que viven estas violencias deben enfrentar su embarazo y la crianza, sin redes de apoyo que acompañen estos nuevos desafíos. Esto implica que, aún cuando se encuentran en procesos de tratamiento para evitar el consumo de drogas, no pueden ejercer el derecho a la maternidad porque los sistemas de protección social desconfían de sus capacidades para asumir el cuidado de sus hijas e hijos. Existen situaciones en que este papel no puede ser cumplido porque efectivamente no existen las condiciones para ello, no obstante, existe un número importante de mujeres, cuyas condiciones familiares y existenciales van transformándose y logran asumirlo.

Desarrollar estrategias de acompañamiento basadas en un enfoque de género y derechos, no solo contribuye a aumentar la adherencia, disminuir las tasas de abandono o aumentar las altas terapéuticas, sino sobre todo, favorece el reconocimiento de las mujeres como titulares de derecho en el marco de un sistema colonial-patriarcal que atraviesa sus historias de vida (Testa \& Spampinato, 2010; Monzeli \& Lopes, 2013; Melo et al., 2014; Grandón, 2017; Portela et al., 2019). Lo anterior requiere comprender las condicionantes socio-demográficas, socioculturales y sanitarias de las mujeres, la singularidad de su experiencia a partir de este contexto socio histórico e identificar las vulneraciones de derechos que viven producto del sistema moderno-colonial-patriarcal. De lo contrario, podemos invisibilizar la violencia estructural que experimentan cotidianamente por el hecho de consumir drogas, ser mujeres, madres, pobres y habitar determinados territorios. 


\section{Acompañamientos en Terapia Ocupacional: Primeras Reflexiones}

Las ideas que presentamos a continuación emergen de una investigación en curso ${ }^{2}$ que estamos desarrollando para caracterizar el modelo de intervención de un Programa Ambulatorio Intensivo de tratamiento para el consumo de drogas y/o alcohol específico para mujeres adultas en embarazo o puerperio del CRS El Pino de la comuna de San Bernardo. Hasta el momento hemos desarrollado nueve entrevistas grupales ${ }^{3}$ (Montero, 2006), dos de las cuales se realizaron al estamento de terapia ocupacional. En este articulo sólo adelantamos las reflexiones preliminares ${ }^{4}$, que hemos ido produciendo a través de la técnica de análisis temático (Díaz, 2018a; Abela, 2000) y el proceso de codificación inicial de dos ejes de análisis: punto de partida de los procesos de acompañamiento y aprendizajes y tensiones.

\section{Punto de Partida de los Procesos de Acompañamiento}

El equipo de terapeutas ocupacionales del Programa PAI-M del Hospital El Pino, está conformado por cuatro profesionales que asumen una posición de habla compartida: se identifican como mujeres, ser feministas y considerar los derechos sexuales y reproductivos como un pilar fundamental de los procesos de acompañamiento, por cuanto reconocer la vivencia de la sexualidad y el derecho a decidir que tienen las mujeres en relación a sus cuerpos es una cuestión de principios. Entienden que en los cuerpos de las mujeres confluyen aspectos subjetivos y condiciones sociales, culturales, políticas y económicas que marcan sus vidas (Quisbert, 2018) y que el maternaje puede ser vivido de manera diversa, cuestionando aquellas visiones universalistas de una "única maternidad": se las acompaña considerando que se trata de maternajes situados e historizados, donde la singularidad exige una lectura crítica de la realidad, considerando el modo en que el sistema patriarcal, colonial y racial se expresa en sus posibilidades e imposibilidades.

Los procesos de acompañamiento se relacionan con la díada madre-hija/o (cuando ella quiere ejercer su maternidad) y se inician desde la gestación, evaluando su situación habitacional y económica, promoviendo el acceso a atenciones obstétricas basadas en el respeto de sus derechos, organizando los espacios cotidianos para la llegada del recién nacida/o y gestionando con la red sociosanitaria los soportes necesarios durante el pre y postparto. Una acción importante es la preparación para el parto apoyando la asistencia a controles y preparando los insumos que se requieren, identificando figuras significativas que puedan brindarles compañía e informando al equipo obstétrico tratante la situación de la gestante, previo consentimiento de la mujer. Muchas de las mujeres del programa, viven altos índices de violencia obstétrica por tener antecedentes de consumo, por ello, en ocasiones se hace un acompañamiento durante el trabajo de parto para resolver dudas del equipo tratante y realizar registros que respalden las condiciones en las que se encuentran y los apoyos que han o no recibido. Cabe destacar que estas acciones son transversales

\footnotetext{
${ }^{2}$ El nombre de esta investigación es Modelo de Intervención con Enfoque de Género Programa Ambulatorio Intensivo de alcohol y/o drogas para mujeres en embarazo y/o post parto. Proyecto InvClínica_DICYT, Código 022091PT_MED. La investigación fue aprobada por el comité de ética de la Universidad y del Hospital El Pino y cumple con todas las consideraciones éticas, incluyendo cartas de consentimiento informado. No hubo gratificaciones ni sanciones que medien la participación.

${ }^{3}$ Del total de entrevistas, 3 corresponden a entrevistas grupales con el equipo (técnicas en rehabilitación, trabajadoras sociales, psicóloga/o, educadora de párvulos, psiquiatras y terapeutas ocupacionales), 4 corresponden a entrevistas grupales por estamentos y 1 entrevista individual al coordinador del programa.

${ }^{4}$ Cabe destacar que los resultados finales serán difundimos de modo amplio una vez concluida la investigación en el formato de artículo de investigación.
} 
dentro del equipo, no obstante, los enfoques de derecho, género y redes adquieren singularidades desde cada campo disciplinar enriqueciendo los procesos de acompañamiento.

Otro aspecto a considerar desde un principio, es que en una sociedad patriarcal, cuando es la mujer quien presenta un consumo problemático, el espacio cotidiano y/o privado se ve cuestionado y se castiga con mayor rigurosidad desde el imaginario social basado en el binarismo de roles. En la experiencia del equipo de terapeutas ocupacionales, las mujeres comienzan a alejarse del rol históricamente asignado y de las labores que como mujer deben cumplir en el espacio privado del hogar, su situación es percibida como fuera de control respecto a lo doméstico y/o laboral, impactando en su confiabilidad e imagen social, ante lo cual priman sentimientos de vergüenza y culpa. Sus parejas habitualmente no asumen los cuidados como parte de su responsabilidad, sus funciones se mantienen fuera de aquel espacio y en caso de haber consumo, la normalización del consumo se relaciona con la comprensión y aceptación de esta conducta como perteneciente al mundo masculino, siendo también señal de masculinidad (Lemus, 2013).

En el periodo de maternaje, durante los primeros 90 días, se realizan acompañamientos en los domicilios, comprendiendo que el puerperio y postparto es un momento de alta fragilidad en el que las mujeres con menor apoyo familiar experimentan un aumento de síntomas asociados a depresión post parto o deseos de consumo; la mantención del vínculo con el recién nacido/a y la experiencia de lactancia materna exclusiva disminuye ambas situaciones. Desde el primer día y durante los primeros meses de vida del recién nacido/a se brinda estimulación temprana en sus hogares o en espacios grupales en concordancia con su vida cotidiana y simultáneamente, se intenta el fortalecimiento de la red familiar para permitir tiempos de relevo y apoyos en los cuidados. Se generan entonces espacios que faciliten el rol de cuidados de sus hijas/os resguardando sus derechos y facilitando que las mujeres que lo deseen puedan cuidar, organizar su vida cotidiana y replantear su proyecto de vida incorporando estrategias de gestión de recursos y gestión de redes de apoyo en el territorio (Pino et al., 2015; Sanz, 2016; Borba et al., 2017; Palacios, 2017; Bianchi, 2018).

En síntesis, los acompañamientos buscan garantizar los derechos de las mujeres y sus hijas/os a partir de un vínculo comprometido que favorece la construcción conjunta del maternaje (comprendiendo que esto puede ser ejercido por varias personas y no solo por la mujer/madre) en sus espacios cotidianas de vida, a través de procesos de acompañamiento sostenido en los territorios y del fortalecimiento de las redes de apoyo, comunicación e intercambio.

\section{Aprendizajes y Tensiones}

Con respecto a los aprendizajes, en primer lugar, se destaca que un acompañamiento comprometido, situado y sostenido en el tiempo, en los espacios cotidianos, favorece los procesos de autodeterminación y autogestión de las mujeres, lo contrario, perpetúa la deprivación social y agudiza las expresiones de violencia (Segato, 2003). En segundo lugar, los procesos de acompañamiento se convierten en prácticas políticas cuando promueven el protagonismo de las mujeres, incluso desde el momento de decidir, de manera voluntaria, si desean ingresar y participar del programa ${ }^{5}$, al generar las condiciones para problematizar

\footnotetext{
${ }^{5}$ Participación comprendida como ingresar de manera voluntaria al programa, acceder a ser evaluada respetando aquellas temáticas que no deseen ser abordadas, asistir a sesiones con diversos miembros del equipo, en caso de no poder asistir acceder a intervenciones domiciliarias que favorezcan el acompańamiento cercano en su proceso terapéutico y lograr objetivos planteados en conjunto con el equipo.
} 
la estigmatización y la violencia estructural que ha marcado sus historias de vida y al establecer un vínculo seguro que apoye la reconstrucción de sus proyectos de vida desde un enfoque de derechos y género. El énfasis del proceso por parte del equipo está en la autonomía de las mujeres y el respeto de sus derechos, comprendiendo que la autonomía sólo es posible cuando existen condiciones para que ellas puedan tomar decisiones respecto de sus cuerpos, sus proyectos, el cuidado de sus hijas/os, entre otros.

Con respecto a las tensiones más importantes se encuentra la necesidad de superar las formas tradicionales de comprender el consumo problemático de drogas y alcohol y, habitualmente asumido desde lógicas medicalizante y patologizantes que instalan formas de razonamiento clínico sobre "casos clínicos" y cuya finalidad es medir el déficit funcional o el nivel de adaptación ocupacional, equilibrio ocupacional o desempeńo ocupacional, jibarizando una compleja trama de acontecimientos históricos a un asunto médicoindividual-funcional. Es necesario desaprender estos mandatos tecnocráticos (Najmanovich, 2012), moderno-coloniales y superar aquellos procesos de estandarización (evaluativo-interventivo) que impiden visibilizar y reconocer la singularidad de los contextos socio-históricos que se materializan en las vidas cotidianas de estas mujeres.

Lo anterior, deriva en comprender la situación de las mujeres no como un problema de salud sino como una problemática social, esto quiere decir, que se debe abordar el consumo problemático de drogas y alcohol pero sin disociarlo de la violencia estructural y las experiencias de opresión y vulneración de derechos a las que han sido expuestas a lo largo de sus vidas. De este modo, los procesos de acompańamiento se pueden convertir en campos de acción situada (Heller, 2002) que pueden ayudar a reconstruir la vida cotidiana (Galheigo, 2003, 2020), pública y privada de las mujeres, desde su reconocimiento y protagonismo, en el marco de las relaciones históricas que las atraviesan.

\section{Reflexiones Finales}

Los procesos de acompañamiento realizados desde un enfoque de derechos y género se presentan como una estrategia fundamental en el Programa ambulatorio intensivo para el consumo de drogas y/o alcohol específico para mujeres en embarazo o puerperio del Hospital El Pino (PAI-M), considerando que este tipo de programas de tratamiento ha sido históricamente pensado para hombres. Si bien el actual escenario nacional muestra que existen una serie de orientaciones desde el Servicio Nacional para la Prevención y Rehabilitación del Consumo de Drogas y Alcohol (SENDA) y desde el Ministerio de Salud (Minsal) sobre la importancia de incorporar los enfoques de derechos y género, aún existe, un abordaje que tiende a homogeneizar las intervenciones sin distinciones de género. Tampoco se incorpora un enfoque de derechos capaz de problematizar la violencia estructural que atraviesa las historias de vida de las mujeres y sus familias, en el marco de un sistema colonial-patriarcal que vulnera sus derechos.

En los análisis preliminares de esta experiencia de investigación, los acompañamientos de terapia ocupacional asumen como punto de partida una lectura crítica de la realidad y una posición de habla compartida, que reconoce a las mujeres como titulares de derecho considerando la díada madre-hija/o desde el periodo de embarazo y maternaje junto a las redes de apoyo que les permiten enfrentar estos nuevos desafíos. En particular, la vivencia de la sexualidad y el derecho a decidir que tienen las mujeres en relación a sus cuerpos es 
considerada una cuestión de principios y el maternaje una experiencia de vida siempre heterogénea, situada e historizada.

Desde las terapias ocupacionales del sur es fundamental asumir un lugar de habla (Ribeiro, 2017) y una postura ética para pensar las jerarquías, las desigualdades, la pobreza, el racismo y el sexismo (Testa, 2012; Monzeli \& Lopes, 2013; Melo et al., 2014; Simó et al., 2016; Palacios, 2017; Grandón, 2017; Díaz, 2018b; Portela et al., 2019; Valderrama, 2019; Díaz-Leiva \& Malfitano, 2021) que viven las mujeres que asisten a este tipo de programas. Develar este lugar es un punto de partida de cualquier acompañamiento, permitiendo una ruptura con el pensamiento moderno colonial que instaura una serie de desigualdades y vulneraciones de derechos que las mujeres y sus redes viven cotidianamente. Problematizar nuestras prácticas y los efectos que tienen en la reproducción del orden patriarcal, reconocer a las mujeres en su dignidad y autonomía y colaborar en la reconstrucción de sus proyectos de vida se transforma en un imperativo.

\section{Referencias}

Abela, J. A. (2000). Las técnicas de análisis de contenido: una revisión actualizada. Granada: Fundación Centro Estudios Andaluces.

Artigas, C. (2005). Una mirada a la protección social desde los derechos humanos y otros contextos internacionales. Santiago: CEPAL.

Bianchi, P. C. (2018). Dos entrecruzamentos da Terapia Ocupacional e o território: reflexões a partir da prática profissional na atenção básica em saúde. Revista Argentina de Terapia Ocupacional, 4(1), 40-46.

Bobes, J., Casas, M., \& Gutiérrez, M. (2011) Manual de Trastornos adictivos. Madrid: Enfoque Editorial.

Borba, P. L. O., Costa, S. L., Savanic, A. C. C., Anastácio, C. C., \& Otae, N. H. (2017). Entre fluxos, pessoas e territórios: delineando a inserçáo do terapeuta ocupacional no Sistema Único de Assistência Social. Cadernos de Terapia Ocupacional da UFSCar, 25(1), 203-214. http://dx.doi.org/10.4322/0104-4931.ctoRE0758.

Consejo Nacional para el Control de Estupefacientes - CONACE. (2007). Mujeres y tratamiento de drogas: guía de asesoría clínica para programas de tratamiento y rehabilitación en drogas en población especifica de mujeres adultas. Santiago: CONACE.

Consejo Nacional para el Control de Estupefacientes - CONACE. (2009). Estrategia Nacional Sobre Drogas 2009-2018. Santiago: CONACE.

Díaz, C. (2018a). investigación cualitativa y análisis de contenido temático. orientación intelectual de revista universum. Revista General de Información y Documentación, 28(1), 119-142.

Díaz, M. (2018b). Terapias ocupacionales: migraciones de saberes y prácticas en Latinoamérica. Revista Ocupación Humana, 18(1), 21-33.

Díaz-Leiva, M. M., \& Malfitano, A. P. S. (2021). Reflexiones sobre las ideas de América Latina y sus contribuciones a las terapias ocupacionales desde el sur. Cadernos Brasileiros de Terapia Ocupacional, 29, e2568. http://dx.doi.org/10.1590/2526-8910.ctoEN1961.

Follegati, L., Troncoso, L., \& Stutzin, V. (2019). Más allá de una educación no sexista: aportes de pedagogías feministas interseccionales: pensamiento educativo. Revista de Investigación Educacional Latinoamericana, 56(1), 1-15.

Galheigo, S. M. (2003). O cotidiano na terapia ocupacional: cultura, subjetividade e contexto históricosocial. Revista de Terapia Ocupacional da Universidade de São Paulo, 14(3), 104-109. http://dx.doi.org/10.11606/issn.22386149.v14i3p104-109.

Galheigo, S. M. (2020). Terapia ocupacional, cotidiano e a tessitura da vida: aportes teórico-conceituais para a construção de perspectivas críticas e emancipatórias. Cadernos Brasileiros de Terapia Ocupacional, 28(1), 5-25. http://dx.doi.org/10.4322/2526-8910.ctoAO2590. 
Grandón, D. (2017). Terapia Ocupacional, discapacidad y género: la interseccionalidad como apertura hacia reflexiones pendientes. Revista Ocupación Humana, 17(2), 34-45.

Guajardo, C. A., \& Galheigo, S. M. (2015). Reflexiones críticas sobre los derechos humanos: contribuciones de la terapia ocupacional latinoamericana. Boletín de la Federación Mundial de Terapeutas Ocupacionales, 71(2), 73-81.

Heller, A. (2002). Sociología de la vida cotidiana. Barcelona: Ediciones Península.

Lemus, A. (2013). Estudio de caso acerca de la incorporación de perspectiva de género en el tratamiento para personas con problemas derivados del consumo de alcohol u otras drogas en un centro de salud mental comunitaria. Santiago: Universidad de Chile.

Lopes, R. E., Malfitano, A. P. S., Silva, C. R., \& Borba, P. L. O. (2014). Recursos e tecnologias em Terapia Ocupacional Social: açóes com jovens pobres na cidade. Cadernos de Terapia Ocupacional da UFSCar, 22(3), 591-602. http://dx.doi.org/10.4322/cto.2014.081.

Lorenzo, S. P. (2009). Drogas y perspectiva de género. plan de atención integral a la salud de la mujer de Galicia: Género y drogas. Galicia: Xunta de Galicia.

Melo, K., Menta, S., \& Serafim, A. (2014). O Programa Bolsa Familia e a questão de gênero: desafios e percepçóes para a atuação do terapeuta ocupacional. Cadernos de Terapia Ocupacional da UFSCar, 22(1), 205-214. http://dx.doi.org/10.4322/cto.2014.022.

Ministerio de Desarrollo Social (2016). Situación de la Pobreza en Chile. Encuesta caracterización socioeconómica Nacional. Chile: Ministerio de Desarrollo Social.

Ministerio de Salud - MINSAL. (2008). Manual de atención personalizada en el proceso reproductivo. Santiago: Minsal.

Ministerio de Salud - MINSAL. (2016). Modelo de gestión de establecimientos hospitalarios. Santiago: Minsal.

Montero, M. (2006). Hacer para transformar: el método en psicología comunitaria. Buenos Aires: Paidós.

Monzeli, G., \& Lopes, R. (2013). Terapia ocupacional e sexualidade: uma revisão nos periódicos nacionais e internacionais da área. Revista de Terapia Ocupacional da Universidade de São Paulo, 23(3), 237-244.

Najmanovich, D. (2012). El mito de la objetividad. Córdoba: Editorial UNRC.

Nebot, M., López, M. J., Ariza, C., Villalbí, J., \& García-Altés, A. (2011). Evaluación de la efectividad en salud pública: fundamentos conceptuales y metodológicos. Gaceta Sanitaria, 25(Supl. 1), 3-8.

Organización Mundial de la Salud - OMS. (2007). Temas de salud e Género. Recuperado el 29 de marzo de 2021, de https:/www.who.int/topics/gender/es/

Palacios, M. (2017). Reflexiones sobre las prácticas comunitarias: aproximación a una Terapia Ocupacional del Sur. Revista Ocupación Humana, 17(1), 73-88.

Parra, D., \& Tortosa, J. (2003). Violencia estructural: una ilustración del concepto. Documentación Social, 131, 1-16.

Pascale, A. (2010). Consumo de drogas durante el embarazo: efectos sobre el binomio materno-fetal, recién nacido y primera infancia. Modalidades terapéuticas y estrategias de prevención. Montevideo: MSP.

Pascale, A. (2015). Consumo de drogas durante el embarazo: efectos sobre el binomio materno-fetal, recién nacido y primera infancia. Modalidades terapéuticas y estrategias de prevención. Montevideo: MSP.

Pino, J., Ceballos, M., \& Sepulveda, R. (2015). Terapia ocupacional comunitaria crítica: diálogos y reflexiones para iniciar una propuesta colectiva. TOG (A Corunã), 12(22), 1-20.

Portela, A., Rosemblat, F., Briglia, J., Rey Ferraro, R., \& Vázquez, N. (2019). encuesta a terapistas ocupacionales mujeres sobre violencia en el ámbito laboral en la argentina. resultados preliminares. Revista Argentina de Terapia Ocupacional, 5(1), 45-59.

Quisbert, M. (2018). La acción colectiva transnacional feminista de la red de salud de las mujeres latinoamericanas y del caribe: politización de los derechos sexuales y reproductivos (Tesis de maestría). Facultad Latinoamericana de Ciencias Sociales, Ecuador.

Ramírez-Elizondo, N. (2011). Enfermería basada en la evidencia, una ruta hacia la aplicación en la práctica profesional. Enfermería en Costa Rica, 32(2), 89-97.

Ribeiro, D. (2017). O que é lugar de fala? Belo Horizonte: Letramento. 
Romero, P., \& Medina-Moreno, C. (1996). Las mujeres adictas: de la descripción a su construcción social. Montevideo: LOM.

Rowlands, M., Miranda, I., \& Arcila, M. (2018). Caracterización de mujeres en embarazo y post parto con consumo de alcohol y sustancias. Santiago: SONEPSYN.

Sanz, S. (2016). Pasos hacia una terapia ocupacional de base comunitaria: una aproximación conceptual al enfoque comunitario en la disciplina en terapia ocupacionales desde el sur derecho, ciudadanía y participación. In S. S. Algado, A. G. Córdoba, O. F. Corrêa, S. M. Galheigo \& S. Garcia Ruzi (Orgs.), Terapias ocupacionales desde el sur: derechos humanos, ciudadanía y participación (pp. 225-243). Chile: Usach.

Segato, R. L. (2003). La Argamasa Jerárquica: Violencia Moral, Reproducción del Mundo y la Eficacia Simbólica del Derecho. Brasília: Serie Antropología.

Servicio Nacional para la Prevención y Rehabilitación del Consumo de Drogas y Alcohol - SENDA. (2009). Estrategia nacional sobre drogas 2009-2018. Santiago: Ministerio del Interior.

Servicio Nacional para la Prevención y Rehabilitación del Consumo de Drogas y Alcohol - SENDA. (2016). Mujeres y tratamiento de alcohol y otras drogas, embarazo, puerperio y lactancia. Santiago: Ministerio del Interior y Seguridad Pública.

Servicio Nacional para la Prevención y Rehabilitación del Consumo de Drogas y Alcohol - SENDA. (2018). SISTRAT, Santiago de Chile. Recuperado el 10 de febrero 2021, de http://sistemas.senda.gob.cl/sistrat/.

Servicio Nacional para la Prevención y Rehabilitación del Consumo de Drogas y Alcohol - SENDA. (2018). Orientaciones técnicas Centros de tratamiento. Santiago: Ministerio del Interior y Seguridad Pública.

Simó, S., Guajardo, A., Oliver, F., Galheigo, S. M., \& Garcia, S. (2016). Terapias ocupacionales desde el sur: derechos humanos, ciudadania y participación. Santiago: Editorial Universidad de Santigo de Chile.

Testa, D. (2012). Aportes para el debate sobre los inicios de la profesionalización de la terapia ocupacional en Argentina. Revista Chilena de Terapia Ocupacional, 12(1), 72-87.

Testa, D., Spampinato, S. (2010). Género, salud mental y terapia ocupacional: algunas reflexiones sobre la influencia de la historia de las mujeres y la perspectiva de género en nuestras prácticas. Rev. Ter. Ocup. Univ. São Paulo, 21(2), 174-181.

Valderrama, C. (2019). Terapias Ocupacionales del Sur: una propuesta para su comprensión. Cadernos Brasileiros de Terapia Ocupacional, 27(3), 671-680. http://dx.doi.org/10.4322/2526-8910.ctoARF1859.

Valencia-Recabarren, P. (2015). Involucramiento parental y consumo de drogas en escolares de Chile. Boletin - Instituto de Estudios de Poblacion y Desarrollo, (19), 1-7.

\section{Contribución de los Autores}

Todas autoras contribuirán igualmente en el desarrollo del artículo y aprobaran su versión final.

Fuente de Financiamiento

Universidad de Santiago de Chile, Usach. Agradecimientos Proyecto InvClínica_DICYT, Código 022091PT_MED,

Vicerrectoría de Investigación, Desarrollo e Innovación.

Autor para la correspondencia

Mónica Matilde Díaz

E-mail: monica.diaz.l@usach.cl

\section{Editor de sección}

Profa. Dra. Carmen Aleida Fernández Moreno 